\title{
Evaluation of hGM-CSF/hTNFa surface- modified prostate cancer therapeutic vaccine in the huPBL-SCID chimeric mouse model
}

Shouhua Lai ${ }^{1 \dagger}$, Zhiyong Huang ${ }^{2 \dagger}$, Yunting Guo ${ }^{1 \dagger}$, Yunqin Cui ${ }^{1}$, Lei Wang ${ }^{1}$, Weifeng Ren ${ }^{1}$, Furong Ying ${ }^{1}$, Hui Gao ${ }^{1}$, Lingxia He${ }^{1}$, Tieli Zhou ${ }^{1 *}$, Jiegen Jiang ${ }^{1 *}$ and Jimin Gao ${ }^{{ }^{*}}$

\begin{abstract}
To validate its efficacy in the context of the human immune system, a novel therapeutic vaccine of hGM-CSF/hTNFa surface-modified PC-3 cells against human prostate cancer was evaluated in the human peripheral blood lymphocytes-severe combined immunodeficiency (huPBL-SCID) chimeric mouse model. The hGM-CSF or/and hTNFa modified vaccines inhibited prostate cancer growth effectively so as to prolong the mouse survival significantly. The splenocytes from the hGM-CSF/hTNFa vaccine-inoculated mice showed the strongest tumor-specific cytotoxicity against PC-3 cells and the highest production of IFNr. These features indicated that type 1 protective immune response was induced efficiently against human prostate cancer and further enhanced through synergetic adjuvant effects of hGM-CSF and hTNFa.
\end{abstract}

Keywords: Cancer immunotherapy, Adjuvant, Synergetic effect, Human immune system, PC-3 prostate cancer cell

\section{Findings}

Prostate cancer is currently the second leading cause of cancer-related death in elderly men and likely develops into androgen-independent at advanced stages, which is refractory to conventional treatments. Cancer vaccine is a rational option for the treatment of androgenindependent prostate cancer [1]. Cancer immunotherapy is getting more and more important, remarkably evidenced by recent checkpoint blockade or chimeric antigen receptor-engineered $\mathrm{T}$ cell-based clinical trials with the impressive efficacy in different types of metastatic cancers $[2,3]$.

Through simultaneous immobilization of streptavidintagged bioactive GM-CSF and TNF $\alpha$ on the biotinylated surface of cancer cells, we previously showed that the resultant cancer vaccines could induce a strong antitumor $\mathrm{T}$ cell immunity [4-7]. In the current study,

\footnotetext{
*Correspondence: wyztli@163.com; jiegen_jiang@yahoo.com; jimingao@yahoo.com

${ }^{\dagger}$ Equal contributors

'Zhejiang Provincial Key Laboratory for Technology and Application of

Model Organisms, School of Laboratory Medicine and Life Sciences,

Wenzhou Medical University, Wenzhou, Zhejiang 325035, China

Full list of author information is available at the end of the article
}

human peripheral blood lymphocytes-severe combined immunodeficiency (huPBL-SCID) model was utilized to mimic the human immune system for evaluating the efficacy of these therapeutic vaccines. The materials and methods used in this study are detailed in Additional file 1.

We demonstrated that SA-hGM-CSF or/and SAhTNF $\alpha$ could be efficiently immobilized on the biotinylated surface of ethanol-fixed PC-3 cells (Additional file 2) and SA-hGM-CSF or/and SA-hTNFa immobilized on the $\mathrm{PC}-3$ cells still retained their bioactivity (Additional file 3).

The PC-3 cells inoculated subcutaneously in nonobese diabetic/severe combined immunodeficiency (NOD/ SCID) mice were found to maintain their original tumorigenicity so as to spread into the blood (Additional file 4). There was no phenomenon of immune leakage [8] in NOD/SCID mice used in this study. The activity of natural killer (NK) cells in the NOD/SCID mice was dramatically reduced by injection of anti-asialo-GM1 antibody (Additional file 5). 
Flow cytometry detected human $\mathrm{CD} 4^{+}, \mathrm{CD}^{+}$, and $\mathrm{CD} 45^{+}$cells in peripheral blood and human $\mathrm{CD} 45^{+}$cells in spleen of huPBL-SCID mice. IHC staining revealed many human $\mathrm{CD}^{+}$and $\mathrm{CD}^{+}$lymphocytes present in spleen and fewer human CD4+ and CD8+ lymphocytes in liver tissue 8 weeks after transfer of huPBL. The results indicated that human $\mathrm{T}$ lymphocytes were successfully engrafted and homed in appropriate lymphoid organs of huPBL-SCID mice (Additional files 6 and 7).

We tested therapeutic effects of different PC-3 cell vaccines modified with hGM-CSF and/or hTNF $\alpha$ on human prostate cancer in the huPBL-SCID mouse model. Compared with other cancer vaccines, the hGMCSF/hTNF $\alpha$ doubly modified cancer vaccine significantly inhibited prostate cancer growth in terms of tumor weight (Fig. 1a) and size (Fig. 1b) and effectively prolonged the mice survival (Fig. 1c).

There were more human leukocytes and CD4+ or CD8+ lymphocytes residing in the lymph nodes in the hGM-CSF/hTNFa doubly modified group than in other groups 8 weeks after vaccination (Additional file 8). Similarly, more human $\mathrm{CD}^{+}$or $\mathrm{CD}^{+} \mathrm{T}$ lymphocytes were found to infiltrate into the tumor tissues in the
hGM-CSF/hTNF $\alpha$ doubly modified group than in other groups (Fig. 2a, b), indicating that the hGM-CSF/hTNFa doubly modified PC-3 cell vaccine could enhance its anti-tumor immunity by increasing the infiltration of human $\mathrm{T}$ lymphocytes into the tumors.

We finally analyzed the tumor-specific cytotoxicity of cytotoxic $\mathrm{T}$ lymphocytes (CTL) in the spleen and revealed that the GM-CSF/TNF $\alpha$ doubly modified vaccine did establish a stronger tumor-specific $\mathrm{T}$ cell immunity than other PC-3 cell vaccines (Fig. 2c). The supernatant from in vitro splenocyte culture in the GM-CSF/TNF $\alpha$ doubly modified group had the highest production of PC-3-specific IFNY (Fig. 2d). Both results indicated that type 1 protective immunity was induced against the human prostate cancer.

Therefore, our current study provided a solid foundation for potential clinical application of this novel hGM-CSF/hTNF $\alpha$ surface-modified prostate cancer therapeutic vaccine. This unique approach can be easily adopted to generate a personalized whole cancer cell vaccine from individual autologous cancer cells, thereby potentially overcoming cancer antigen heterogeneity [9-11].

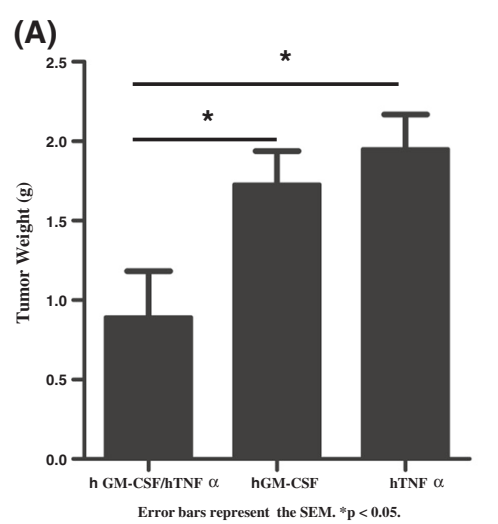

(B)

(C)

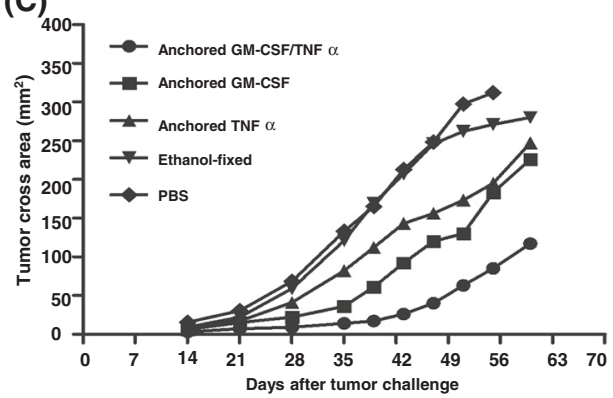

Fig. 1 Therapeutic effects of hGM-CSF or/and hTNFa modified PC-3 cell vaccines on human prostate cancer in the huPBL-SCID mouse model. After inoculation with PC-3 prostate cancer cell vaccine, huPBL-SCID mice were treated i.p. with PC-3 cancer cell vaccines or PBS on days 0, 7, and 14. We collected and weighted the tumors in the mice died at different time points or those still living on day $60(p<0.05)$. There were no data at the time point on day 60 in the PBS control group because all mice died before/on day 56 (a). In addition, we drew a curve of the mean size of cross-sectional area of the tumors in each group along with the time period (b). Meanwhile, we recorded the time of all mice death with the day of injecting PC-3 cancer cells as the starting point and drew survival curves $(p<0.05)(\mathbf{c})$. The results represented one of three separate experiments 


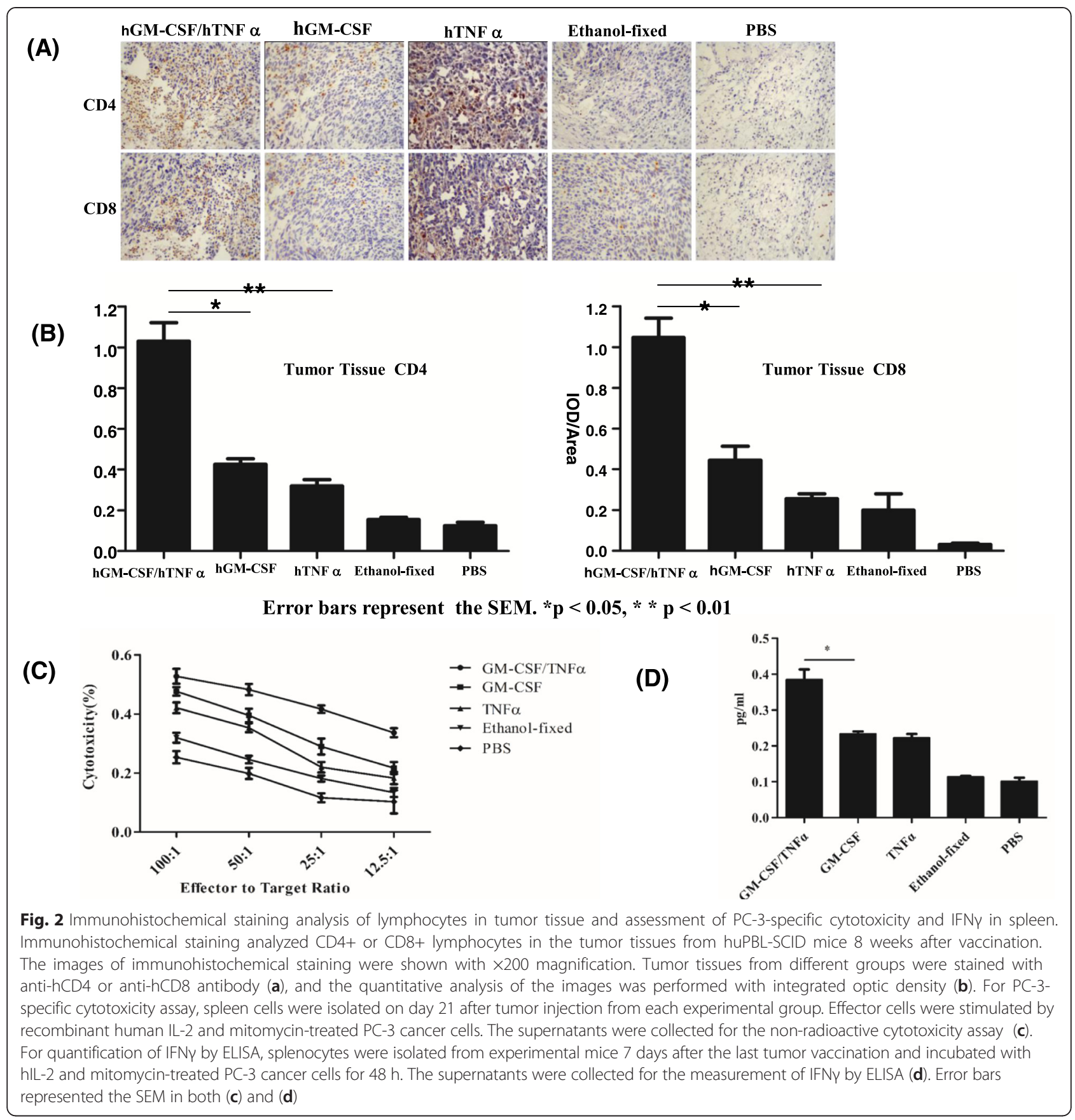

\section{Additional files}

Additional file 1: Materials and methods.

Additional file 2: Flow cytometric analysis of the modification efficiency of streptavidin (SA)-tagged hGM-CSF and/or hTNFa on the surface of ethanol-fixed and biotinylated PC-3 prostate cancer cell vaccine. Ethanol-fixed PC-3 cells were biotinylated and then incubated with SA-hGM-CSF, SA-hTNFa, or both as described before. The presence of hGM-CSF or/and hTNFa was assessed with allophycocyanin (APC)-labeled anti-hGM-CSF (1:400) or/and fluorescein isothiocyanate (FITC)-labeled anti-hTNFa (1:100) monoclonal antibodies for flow cytometric analysis. Ethanol-fixed PC-3 cells were used as control.
Additional file 3: Bioactive assays of hGM-CSF and hTNFa immobilized on the surface of ethanol-fixed PC-3 prostate cancer cell vaccine. Membrane fractions were prepared from the hGM-CSF/ hTNFa doubly modified PC-3 cancer cell vaccine as described in materials and methods. (A) Bioactive assay of hGM-CSF immobilized on the surface of ethanol-fixed PC-3 cell vaccine. The proliferative activity of membrane-conjugated hGM-CSF was assessed on TF-1 cells with SA-hGM-CSF/SA-hTNFa un-modified PC-3 cell vaccine as negative control. (B) Bioactive assay of hTNF-a immobilized on the surface of ethanol-fixed PC-3 cell vaccine. The cytotoxic activity of membraneconjugated hTNFa was assessed on L929 cells with hGM-CSF/hTNFa un-modified PC-3 cell vaccine as negative control. Data were shown as the mean \pm SEM of triplicates. 
Additional file 4: Flow cytometric analysis of tumor tissue and peripheral blood from NOD/SCID mice with anti-hPSMA and anti-HLA. Tumor tissues and peripheral blood were isolated on the 30th and 60th day to prepare single cell suspensions. The presence of PSMA and HLA (human leukocyte antigen) was assessed with APC-labeled anti-hPSMA (1:100) and FITC-labeled anti-hHLA (1:200) monoclonal antibodies, respectively, for flow cytometric analysis. The LNCaP prostate cancer cells were used as a positive control because they could secrete PSA and express PSMA. (A) The single cell suspension from tumor tissue on day 30 with APC-labeled anti-hPSMA. (B) The LNCaP prostate cancer cells. (C) The single cell suspension from tumor tissue on day 30 with FITC-labeled anti-hHLA. (D) The blood from PC-3 inoculated NOD/ SCID mice on day 30 with FITC-labeled anti-hHLA. (E) The white cells from PC-3 inoculated NOD/SCID mice on day 60 with FITC-labeled anti-hHLA.

Additional file 5: Screen for the immune "leakage" phenomenon of NOD/SCID mice and detection of the levels of NK cells before and after injection of NK cell inhibitor GM1. (A) Concentration of lgG in peripheral blood serum of NOD/SCID mice. The immune "leakage" phenomenon was screened by the levels of IgG through ELISA kit (Beyotime) in $25 \mathrm{NOD} / \mathrm{SCID}$ mice with standard and blank controls (data from the controls not shown). The concentration of IgG lower than $10 \mu \mathrm{g} / \mathrm{ml}$ was defined as no immune leakage. (B) The changes of CD122 levels in peripheral blood of NOD/SCID mice before and after injection of NK cell inhibitor anti-asialo-GM1 antibody. CD122 was assessed with FITC-labeled anti-CD122 (1:200) monoclonal antibody for flow cytometric analysis. Left: CD122 level before injection of NK cell inhibitor antiasialo-GM1 antibody; middle: CD122 level after injection of NK cell inhibitor anti-asialo-GM1 antibody; right: comparison of CD122 levels before and after injection of NK cell inhibitor anti-asialo-GM1 antibody.

Additional file 6: Flow cytometric analysis of human lymphocytes in the peripheral blood and spleen tissue of huPBL-SCID mice after huPBL transplantation. (A) The presence of $\mathrm{CD}_{4} 5^{+}, \mathrm{CD} 8^{+}$, or $\mathrm{CD} 4^{+}$cells was assessed respectively with phycoerythrin (PE)-labeled anti-hCD45, PE-Cy7-labeled anti-hCD8, and PE-Cy7-labeled anti-hCD4 monoclonal antibodies for flow cytometric analyses. (B) Flow cytometric analysis of human $\mathrm{CD} 45^{+}$cells in the spleen tissue of huPBL-SCID mice 8 weeks after huPBL transplantation. Spleen was isolated from huPBL-SCID 8 weeks after huPBL transplantation and single cell suspensions were prepared from the isolated spleen. The presence of $\mathrm{CD} 45^{+}$cells was assessed with PE-labeled anti-hCD45 monoclonal antibody through a flow cytometer. NOD/SCID mice without huPBL transplantation were used as the negative controls.

Additional file 7: Immunohistochemical staining of spleen and liver tissues from huPBL-SCID mice 8 weeks after huPBL transplantation. The images of immunohistochemical staining were shown with $\times 200$ magnification. The spleen and liver tissues from huPBL-SCID mice were stained with anti-hCD4 or anti-hCD8 antibody (A), and the quantitative analysis of the images was performed with integrated optic density (B). The spleen and liver tissues from NOD/SCID mice without huPBL transplantation were used as negative controls.

Additional file 8: Immunohistochemical staining analysis of the lymph node tissues from huPBL-SCID mice 8 weeks after vaccination. The images of immunohistochemical staining were shown with $\times 200$ magnification. Lymph node tissues from different groups were stained with rat anti-hCD45 (A), anti-hCD4 (B), or anti-hCD8 antibody (C). The quantitative analysis of those images was carried out with integrated optic density (D)

\section{Abbreviations}

hGM-CS: human granulocyte-macrophage colony-stimulating factor; hTNFa: human tumor necrosis factor a; SA: streptavidin; huPBL: human peripheral blood lymphocytes; IFN: interferon; NOD/SCID: nonobese diabetic/ severe combined immunodeficiency; PSA: prostate-specific antigen; PSMA: prostate-specific membrane antigen; NK: natural killer; PBS: phosphate-buffered saline; ELISA: enzyme-linked immunosorbent assay; FITC: fluorescein isothiocyanate; HLA: human leukocyte antigen; APC: allophycocyanin; PE: phycoerythrin.

\section{Competing interests}

The authors declare that they have no competing interests.

\section{Authors' contributions}

$S L$ carried out the vaccine preparation and animal studies, participated in the bioactive assays and immunologic reconstitution, established the tumor models, and drafted the manuscript. ZH carried out tumor-specific cytotoxicity assay and histological and immunohistochemical analysis and participated in establishment of the tumor models. YG participated in the bioactive assays and immunologic reconstitution and helped to draft the manuscript. YC carried out the ELISA. WR participated in the NOD/SCID mouse preparation. LW participated in the design of the study. FY performed the statistical analysis. HG carried out cell culture. LH participated in the animal studies. TZ designed animal studies and participated in the manuscript preparation. JJ participated in the study design and helped to draft the manuscript. JG conceived of the study, participated in its design and coordination, and helped to draft the manuscript. All authors read and approved the final manuscript.

\section{Acknowledgements}

This work was supported partly by Chinese National 863 plan (2012AA02A407), The Scientific Research Fund of Ministry of Public Health (201231029), Zhejiang Provincial Major Research Program (2010C13007), Key Science and Technology Innovation Team of Zhejiang Province, the Natural Science Foundation of Zhejiang Province (Y2110580, LY12C07001,

LY13H100003, LZ14H260001), Zhejiang Provincial Program for the Cultivation of High-level Innovative Health Talents, Science and Technology Innovation Program for College/University Students in Zhejiang Province (2012R413039, 2012R413041).

\section{Author details}

'Zhejiang Provincial Key Laboratory for Technology and Application of Model Organisms, School of Laboratory Medicine and Life Sciences, Wenzhou Medical University, Wenzhou, Zhejiang 325035, China. ${ }^{2}$ Department of Cardiothoracic Surgery, Nanfang Hospital, Southern Medical University, Guangzhou, Guangdong 510515, China.

Received: 19 May 2015 Accepted: 16 June 2015

Published online: 25 June 2015

\section{References}

1. Simons JW. Prostate cancer immunotherapy: beyond immunity to curability. Cancer Immunol Res. 2014;2:1034-43.

2. Shi L, Chen S, Yang L, Li Y. The role of PD-1 and PD-L1 in T-cell immune suppression in patients with hematological malignancies. J Hematol Oncol. 2013;6:74.

3. Han E, Li X, Wang C, Li T, Han S. Chimeric antigen receptor-engineered T cells for cancer immunotherapy: progress and challenges. J Hematol Oncol. 2013;6:47.

4. Gao J, Huang S, Li M, Luo R, Wang X, Takashima A. GM-CSF-surface-modified B16.F10 melanoma cell vaccine. Vaccine. 2006;24:5265-8.

5. Hu Z, Tan W, Zhang L, Liang Z, Xu C, Su H, et al. A novel immunotherapy for superficial bladder cancer by intravesical immobilization of GM-CSF. J Cell Mol Med. 2010;14:1836-44.

6. Zhang X, Shi X, Li J, Hu Z, Zhou D, Gao J, et al. A novel therapeutic vaccine of mouse GM-CSF surface modified MB49 cells against metastatic bladder cancer. J Urol. 2012;187:1071-9.

7. Yin W, He Q, Hu Z, Chen Z, Mao Q, Sun Z, et al. A novel therapeutic vaccine of GM-CSF/TNFalpha surface-modified RM-1 cells against the orthotopic prostatic cancer. Vaccine. 2010;28:4937-44.

8. Bankert RB, Hess SD, Egilmez NK. SCID mouse models to study human cancer pathogenesis and approaches to therapy: potential, limitations, and future directions. Front Biosci. 2002;7:c44-62.

9. Boyd LK, Mao X, Lu YJ. The complexity of prostate cancer: genomic alterations and heterogeneity. Nat Rev Urol. 2012;9:652-64.

10. Spans L, Clinckemalie L, Helsen C, Vanderschueren D, Boonen S, Lerut E, et al. The genomic landscape of prostate cancer. Int J Mol Sci. 2013;14:10822-51.

11. Wyatt AW, Mo F, Wang Y, Collins CC. The diverse heterogeneity of molecular alterations in prostate cancer identified through next-generation sequencing. Asian J Androl. 2013;15:301-8. 